\title{
Ultrastable nanogold catalyst-on the way going to practical application
}

\author{
Masatake Haruta*
}

The catalytic performance of gold nanoparticles (NPs) can be dramatically changed by choosing support materials and by designing the fine architecture of contact structure between gold NPs and the supports. So far many kinds of base metal oxides including acidic metal oxides such as $\mathrm{Nb}_{2} \mathrm{O}_{5}$ [1], carbon materials, organic polymers have been reported as effective support for gold NPs. Each one of supported gold NPs show unique catalytic activity and selectivity. A typical example is core/shell structured $\mathrm{Au} / \mathrm{NiO}$, by which a new chemical plant to produce methylmethacrylate from methacrolein was constructed in 2008. Other examples are $\mathrm{AsH}_{3}$ gas sensors and mercury collectors for the environmental analyses. Accordingly, a key current issue is stability and life of gold catalysts. Typical examples for long life catalysts are MINTEK catalysts [2] and YD-3 Yantai catalysts, the latter is composed of $\mathrm{Al}_{2} \mathrm{O}_{3}$ modified with $\alpha-\mathrm{Fe}_{2} \mathrm{O}_{3}, \mathrm{La}_{2} \mathrm{O}_{3}$ and gold NPs [3].

Recently, a team of DICP directed by J. Wang and T. Zhang discovered that $\mathrm{Au}$ NPs could interact strongly with hydroxyapatite (HAP, $\mathrm{Ca}_{10}\left(\mathrm{PO}_{4}\right)_{6}(\mathrm{OH})_{2}$, the main component of bones and teeth) support to form the so called Strong Metal-Support
Interaction (SMSI) upon high-temperature calcination [4]. The SMSI results in the encapsulation of Au NPs which improves their sintering resistance significantly but reduces their activity due to the coverage of the active sites.

Quite recently, the authors have modified the HAP by incorporating $\mathrm{TiO}_{2}$ into the HAP support [5]. Using this support they developed a new type of $\mathrm{Au} / \mathrm{HAP}-\mathrm{TiO}_{2}$ catalyst in which the $\mathrm{Au}$ NPs were partially covered by a thin layer of the HAP and partially exposed and contacted with $\mathrm{TiO}_{2}$. By this unique nanoscale architectural design, it was found that this supported $\mathrm{Au}$ nanocatalyst not only possessed high activity and good sintering resistance after calcination at $800{ }^{\circ} \mathrm{C}$ for a variety of reactions but also demonstrated excellent durability that outperforms a commercial TWC (Three Way Catalyst) for CO oxidation under simulated practical conditions.

This work will provide a new avenue for practical applications of supported Au nanocatalysts, especially for high temperature catalytic reactions, thus may facilitate the practical applications or even commercialization of supported Au catalysts.

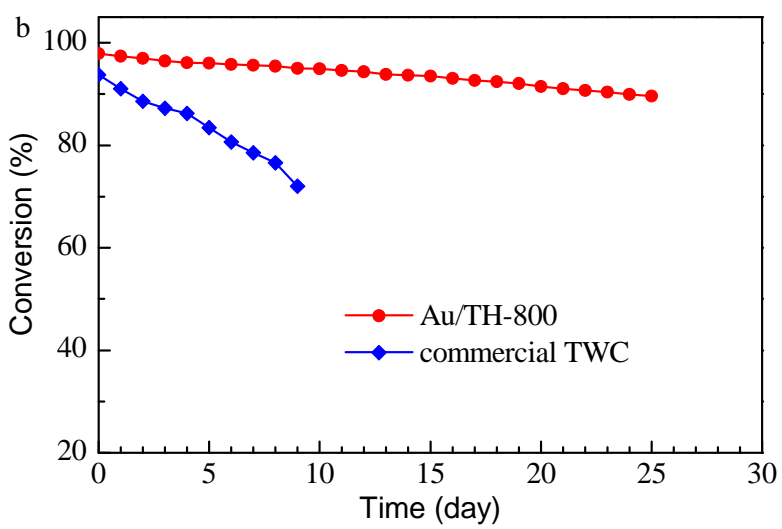

Fig. 1. (a) CO oxidation curves of Au/X-800. Gas flow: $1 \mathrm{v} \% \mathrm{CO}+1 \mathrm{v} \% \mathrm{O}_{2}$ and balanced with He, $33.3 \mathrm{~mL} \mathrm{~min} \mathrm{~m}^{-1}, 100 \mathrm{mg}$ catalyst, space velocity (SV) =

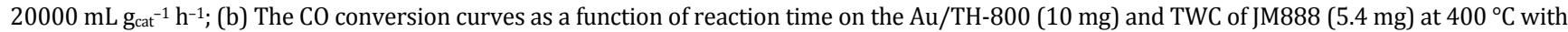
$\mathrm{SV}$ of $200 \mathrm{~L} \mathrm{~g}_{\mathrm{cat}}{ }^{-1} \mathrm{~h}^{-1}$ and $1100 \mathrm{~L} \mathrm{~g}_{\mathrm{cat}}{ }^{-1} \mathrm{~h}^{-1}$, respectively. Reaction gas composition: $1.6 \mathrm{v} \% \mathrm{CO}, 1 \mathrm{v} \% \mathrm{O}_{2}, 0.01 \mathrm{v} \%$ propene, $0.0087 \mathrm{v} \%$ toluene, $10 \mathrm{v} \%$ water and balanced with He. Reproduced with permission from Wiley. 


\title{
Graphical Abstract
}

Chin. J. Catal., 2016, 37: 1441-1442 doi: 10.1016/S1872-2067(16)62526-3

Ultrastable nanogold catalyst-on the way going to practical application

Masatake Haruta *

Tokyo Metropolitan University, Japan; Dalian Institute of Chemical Physics, Chinese Academy of Sciences, China

In the SMSI effect tuned catalyst, $\mathrm{Au} / \mathrm{TiO}_{2}-\mathrm{HAP}$, the $\mathrm{Au}$ NPs are located at the $\mathrm{TiO}_{2} / \mathrm{HAP}$ interfaces, forming anchored and partially covered Au NPs which are ultrastable and highly active for various high temperature reactions.

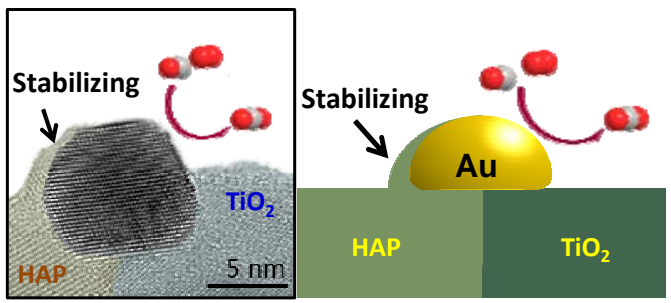

Masatake Haruta

Research Center for Gold Chemistry \& Department of Applied Chemistry, Graduate School of Urban Environmental Sciences, Tokyo Metropolitan University, 192-0397, Japan

Visiting Professor for Senior International Scientists of Chinese Academy of Sciences

Gold Catalysis Research Center \& State Key Laboratory of Catalysis, Dalian Institute of Chemical Physics, Chinese Academy of Sciences, Dalian, 116023, China

Tel/ Fax: +81-42-677-2852

E-mail: haruta-masatake@center.tmu.ac.jp

Received 19 August 2016; Accepted 22 August 2016

Published 5 September 2016
DOI: $10.1016 / \mathrm{S} 1872-2067(16) 62526-3$

\section{References}

[1] T. Murayama, M. Haruta, Chin. J. Catal., 2016, in press.

[2] J. S. McPherson, D. T. Thompson, THE STREM CHMIKER, 2009, XXIV, 21-24.

[3] C. Qi, S. Zhu, H. Su, H. Lin, R. Guan, Appl. Catal. B, 2013, 138-139, 104-112.

[4] H. Tang, J. Wei, F. Liu, B. Qiao, X. Pan, L. Li, J. Liu, J. Wang, T. Zhang, J. Am. Chem. Soc., 2016, 138, 56-59.

[5] H. Tang, F. Liu, J. Wei, B. Qiao, K. Zhao, Y. Su, C. Jin, L. Li, J. Liu, J. Wang, T. Zhang, Angew. Chem. Int. Ed., 2016, 55, 10606-10611.

\section{超稳纳米金催化剂一走在实用化的路上}

\author{
Masatake Haruta \\ a首都大学东京, 城市环境科学研究生院金化学和应用化学研究中心, 东京192-0397, 日本 \\ b中国科学院大连化学物理研究所金催化研究中心，辽宁大连116023
}

纳米金的催化性能受载体影响巨大, 选择合适的载体或设计金属一载体界面精细结构能显著影响纳米金的催化性能. 迄今发现各种载体包括酸、碱金属氧化物、碳材料以及有机聚合物均可作为纳米金的有效载体. 相应的各种金催化剂均 展现出独特的催化活性与选择性.一个典型的例子是核壳结构的 $\mathrm{Au} / \mathrm{NiO}$ 催化剂, 基于该催化剂催化异丁烯醛制备异丁烯 酸甲酯的化工厂已于2008年开始兴建. 金催化剂在 $\mathrm{AsH}_{3}$ 气体传感器和录收集器等环境分析方面也开始实际应用. 因而, 金 催化剂的稳定性和使用寿命成为当前关注的焦点问题. 目前报道的长寿命金催化剂典型例子有MINTEK催化剂和YD-3烟 台催化剂, 后者是由 $\alpha-\mathrm{Fe}_{2} \mathrm{O}_{3}$ 和 $\mathrm{La}_{2} \mathrm{O}_{3}$ 改性氧化铝负载的金催化剂.

中国科学院大连化学物理研究所张涛院士和王军虎研究员团队在近期研究中发现高温焙烧条件下 $\mathrm{Au}$ 纳米颗粒与羟基 磷灰石(HAP)载体之间会发生金属-载体强相互作用(Strong Metal-Support Interaction简称SMSI)效应. SMSI效应导致载体 对Au纳米颗粒形成包裹, 可以有效提升Au纳米颗粒的抗烧结性能, 但其对活性位的覆盖也会导致催化剂活性的下降.

最近, 该团队通过向载体 $\mathrm{HAP}$ 中添加 $\mathrm{TiO}_{2}$ 进行修饰, 成功设计开发出 $\mathrm{Au} / \mathrm{HAP}-\mathrm{TiO}_{2}$ 催化剂. 该催化剂上 $\mathrm{Au}$ 纳米颗粒与 $\mathrm{HAP}$ 接触的一侧被 $\mathrm{HAP}$ 薄层包裹, 与 $\mathrm{TiO}_{2}$ 接触的一侧裸露, 呈现出独特的半包裹结构. 通过这种纳米尺度的结构设计, 该金 催化剂经过 $800^{\circ} \mathrm{C}$ 的高温焙烧后不仅对一系列反应均表现出可观的催化活性和优异的抗烧结性能, 且在模拟汽车尾气CO 消除反应中表现出优于商业三效催化剂的反应稳定性. 该工作为负载型纳米金催化剂的应用, 特别是在高温催化反应中 的实际应用提供了新途径, 因此有望促进负载型金催化剂的实用化乃至商业化进程.

关键词: 金属-载体强相互作用; 纳米金催化剂; 超稳定性; 高活性

收稿日期: 2016-08-19. 接受日期: 2016-08-22. 出版日期: 2016-09-05.

*通讯联系人. 电话/传真: +81-42-677-285; 电子信箱: haruta-masatake@center.tmu.ac.jp

本文的英文电子版由Elsevier出版社在ScienceDirect上出版(http://www.sciencedirect.com/science/journal/18722067). 